\title{
Erratum to: All-Inside Meniscal Repair: Updates on Technique
}

Sergio Rocha Piedade, Rodrigo Pereira da Silva Nunes, Camila Cohen Kaleka, and Tulio Pereira Cardoso

\section{Erratum to:}

Chapter 9 in: R.F. LaPrade et al. (eds.),

The Menisci, DOI 10.1007/978-3-662-53792-3_9

In the original version of chapter 9, the chapter author's last name was wrongly given as Cardoso, but it should be given as Pereira Cardoso. The name of the author should be read as Tulio Pereira Cardoso. 